\title{
Protrusio acetabular en artroplastía total de cadera: diez tácticas para un buen resultado quirúrgico
}

\section{Total Hip Arthroplasty in Protrusio Acetabuli: Ten Tips to Improve Surgical Outcomes}

\author{
Francisco Bengoa1(1) Alberto López ${ }^{2,3(0)}$ David Dabed ${ }^{2,4(0)}$ Nicolas Rojas $2,5(0$ \\ Claudio Diaz-Ledezma ${ }^{4,6}$
}

${ }^{1}$ Departamento de Ortopedia, University of British Columbia,

Address for correspondence Francisco Bengoa, MD, Department of Vancouver, British Columbia, Canada

${ }^{2}$ Clínica RedSalud Santiago, Santiago, Chile

${ }^{3}$ Hospital Barros Luco Trudeau, Santiago, Chile

${ }^{4}$ Hospital El Carmen - Dr. Luis Valentín Ferrada, Santiago, Chile

${ }^{5}$ Instituto Traumatológico Dr. Teodoro Gebauer Weisser, Santiago, Chile

${ }^{6}$ Clínica Las Condes, Santiago, Chile

Rev Chil Ortop Traumatol 2021;62(2):e127-e135.

\section{Resumen \\ Palabras Clave \\ - protrusio acetabular \\ - protrusio \\ - artroplastía total de cadera \\ - ATC}

Abstract
Keywords
- protrusio acetabuli
- protrusio
- total hip arthroplasty
- THA

Los pacientes candidatos a artroplastía total de cadera con protrusio acetabular asociada generan distintos desafíos en los equipos quirúrgicos. Múltiples estrategias han sido utilizadas a lo largo de los años para optimizar los resultados. Mediante una revisión de la evidencia actualizada disponible, proponemos diez tácticas a realizar en el manejo de estos pacientes que pueden mejorar y hacer predecible el tratamiento de un paciente con protrusio acetabular al que se le realiza una artroplastía total de cadera.

Nivel de Evidencia V.

Patients with acetabular protrusio and osteoarthritis are a challenge for the surgical team. Many strategies have been developed to anticipate, plan and optimize the surgical results of these patients. Based on the current available clinical evidence, we propose ten tips to improve the surgical management of hip arthroplasty patients with protrusio acetabuli.

Level of Evidence $V$.

\section{Introducción}

La deformidad acetabular denominada protrusión acetabular, también conocida como protrusio acetabuli, artrokatadisis, o pelvis de Otto, está presente cuando la porción medial de la cabeza femoral sobrepasa la línea ilioisquiática. Esto determina la medialización del centro de rotación de la cadera (COR, por sus siglas en inglés), e impone ciertos desafíos que hacen que la cirugía de reemplazo articular de cadera en estos casos sea recibido

07 de mayo de 2020

aceptado

21 de enero de 2021
DOI https://doi.org/

10.1055/s-0041-1735576. ISSN 0716-4548. (c) 2021. Sociedad Chilena de Ortopedia y Traumatologia. All rights reserved.

This is an open access article published by Thieme under the terms of the Creative Commons Attribution-NonDerivative-NonCommercial-License, permitting copying and reproduction so long as the original work is given appropriate credit. Contents may not be used for commercial purposes, or adapted, remixed, transformed or built upon. (https://creativecommons.org/ licenses/by-nc-nd/4.0/)

Thieme Revinter Publicações Ltda., Rua do Matoso 170, Rio de Janeiro, RJ, CEP 20270-135, Brazil 
considerada un procedimiento tipo "prótesis primaria dificil".

Las características anatómicas y el tipo de paciente en que se presenta el protrusio acetabular hacen que el cirujano deba considerar varias particularidades a lo largo de la evaluación y tratamiento. El objetivo de esta revisión narrativa es presentar diez tácticas que contribuyen a que el tratamiento se realice de manera predecible y exitosa.

1. Si el paciente no tiene un diagnóstico reumatológico asociado a protrusio acetabular, ¿debo considerarlo un caso idiopático y operarlo, o debo estudiar otras enfermedades asociadas previo a la cirugía?

La protrusión acetabular es una condición infrecuente, que se presenta de manera primaria (idiopática) y secundaria a variadas patologías, tales como enfermedades reumatológicas, infecciosas, metabólicas etc. ( - Tabla 1). En pacientes sin un diagnóstico reumatológico previo, pero en quienes el cirujano tiene la duda razonable, una opción válida es aplicar un cuestionario de tamizaje de estas patologías. Nuestra recomendación es el cuestionario de Barbour et al., ${ }^{1}$ que utiliza varias preguntas clínicas y sólo dos parámetros de laboratorio, como la velocidad de eritrosedimentación (VHS) y el factor reumatoideo (-Tabla 2). Un puntaje de 3 o más es $97 \%$ sensible para patología reumatológica, y hace perentoria una derivación al especialista.

En relación a la concomitancia de osteomalacia y protrusio acetabular reportada ya hace varias décadas, es altamente recomendable solicitar valores de vitamina D en pacientes que se van a someter a cirugía, especialmente considerando que existen publicaciones que sugieren estudio de hipovitaminosis $\mathrm{D}$ en todos los pacientes candidatos a artroplastia. ${ }^{2,3}$

No es clara la utilidad de estudio de tuberculosis $u$ otros patógenos a través de muestras tomadas durante la cirugía. En relación a las otras causas, parecieran ser tan poco frecuentes, y algunas de ellas, tan clínicamente evidentes, que sugerimos no realizar estudios particulares preoperatorios. La forma idiopática es diagnosticada en pacientes en los que no se encuentra factores causales. Es un diagnóstico de exclusión, pero, pese a ello, es el más frecuente.

2. Si el paciente tiene un diagnóstico reumatológico como causa de protrusio acetabular, ¿cuál es el mejor momento para realizar la cirugía?

Si el protrusio acetabular es secundario a patología reumatológica, el trabajo en conjunto con el reumatólogo tratante es crucial. En aquellos casos cuyo control reumatológico ha sido abandonado, recomendamos no realizar la cirugía hasta una evaluación formal por un especialista de esa área.

Los pacientes con enfermedades reumatológicas tienen más riesgo de complicaciones en cirugía de reemplazo articular. $^{4,5}$ Por este motivo, la optimización de sus comorbilidades (anemia, malnutrición etc.), así como el correcto manejo de los fármacos inmunosupresores son clave en la prevención de complicaciones perioperatorias.

Tabla 2 Cuestionario de enfermedad inflamatoria articular de Barbour et al. ${ }^{1}$

\begin{tabular}{|l|}
\hline Rigidez matinal mayor que 1 hora \\
\hline $\begin{array}{l}\text { Distribución característica para una enfermedad } \\
\text { inflamatoria articular }\end{array}$ \\
\hline $\begin{array}{l}\text { Familiar de primer grado con una enfermedad inflamatoria } \\
\text { articular }\end{array}$ \\
\hline Evidencia clínica de sinovitis \\
\hline $\begin{array}{l}\text { Velocidad de eritrosedimentación } \geq 20 \text { en hombres } \\
\text { o } \geq 30 \text { en mujeres }\end{array}$ \\
\hline Factor reumatoideo positivo $(\geq 1 / 80)$ \\
\hline Erosiones en radiografías de pies o manos \\
\hline $\begin{array}{l}\text { Mejoría obtenida con antiinflamatorios no esteroidales o } \\
\text { corticoides }\end{array}$ \\
\hline
\end{tabular}

Nota: Para un puntaje de 3 o más, este cuestionario presenta una sensibilidad de $97 \%$, especificidad de $55 \%$, valor predictivo positivo de $49 \%$ y valor predictivo negativo de $97 \%$.

Tabla 1 Causas de protrusio acetabular secundario

Infecciosas
Neisseria gonorrhoeae, echinococcus spp., staphylococcus spp., streptococcus spp., mycobacterium tuberculosis

\section{Neoplásicas}

Hemangioma, metastásicas (mama, próstata), neurofibromatosis, osteonecrosis inducida por radiación

Inflamatorias

Artritis reumatoide, espondilitis anquilosante, artritis reumatoide juvenil, artritis psoriática, condrolisis idiopática aguda, síndrome de Reiter, osteolisis secundaria a artroplastía

Metabólicas

Enfermedad de Paget, osteogénesis imperfecta, ocronosis, acrodisostosis, osteomalacia, hiperparatiroidismo

\section{Traumáticas}

Secuela de fractura de acetábulo, iatrogénica tras artroplastía

\section{Genéticas}

Síndrome tricorrinofalángicos, trisomía 18, síndrome de Sticker, síndrome de Ehler Danlos, síndrome de Marfan, enfermedad de células falciformes 
Probablemente el documento más importante respecto al manejo de fármacos inmunosupresores en pacientes candidatos a artroplastía es la guía conjunta de la American Association of Hip and Knee Surgeons y el American College of Rheumatology. ${ }^{6}$ Si bien cada paciente debe ser evaluado de forma individual, discutiendo las alternativas con el reumatólogo tratante, las recomendaciones internacionales se pueden resumir en:

I. Evaluar el grado de actividad de enfermedad inflamatoria:

Idealmente, el paciente debe estar en remisión u oligosintomático, con un esquema de tratamiento ya establecido. Sin embargo, es destacable que, cuando se entrevistó a un panel de pacientes reumatológicos sometidos a artroplastía respecto a la relevancia de las reactivaciones de la enfermedad (manejado a través de fármacos) versus el riesgo de infección, éstos dieron más relevancia a la disminución del riesgo de infección. Con esto se puede inferir que la mayoría de los pacientes estarían de acuerdo con modificar su esquema terapéutico (aunque este reactive los síntomas) siempre y cuando esta intervención disminuya el riesgo de infección. ${ }^{7}$ Además, es relevante evaluar el riesgo cardiovascular de estos pacientes, que puede verse elevado. ${ }^{8}$

\section{Modificación de fármacos inmunomoduladores:}

\section{a. Glucocorticoides:}

Existe una asociación dosis-respuesta, con aumento del riesgo de complicaciones con dosis de prednisona $>15 \mathrm{mg}$ al día, por lo que aquellos pacientes que utilizan dosis mayores debieran considerarse con su enfermedad no controlada y diferir la cirugía hasta lograr dosis menores de corticoides.

Dosis de hasta $15 \mathrm{mg}$ de prednisona (o equivalentes) idealmente $<10 \mathrm{mg}$, se consideran seguras, y no requieren suspensión en el período perioperatorio.

En cuanto al uso de "dosis de stress" en el perioperatorio, se ha evidenciado un mayor riesgo de infección con estas dosis suprafisiológicas, sin beneficio "hemodinámico" comparado con no administrar, por lo que no se recomienda en la actualidad. ${ }^{9}$

b. Fármacos antirreumáticos modificadores de la enfermedad (DMARD por su sigla en inglés incluyen metotrexato, leflunomida, hidroxicloroquina $\mathrm{y}$ sulfasalazina):

Metanálisis, incluyendo pequeños estudios randomizados, ${ }^{10,11}$ no han evidenciado un aumento de riesgo de infección al mantener estos fármacos, pero sí un mayor riesgo de crisis inflamatorias al suspenderlos, por lo cual se deben mantener en el perioperatorio sin cambios.

\section{c. Biológicos:}

Tienen un riesgo aumentado de infecciones tanto del sitio quirúrgico como de otros sistemas, por lo cual se sugiere suspender y programar la cirugía al término del ciclo de cada fármaco específico. Por ejemplo, infliximab, cuya administración es cada cuatro semanas, debiera omitirse la dosis de la semana cuatro y operarse en la semana cinco, a modo de encontrarse en el nadir del efecto inmunosupresor.

Se recomienda diferir el reinicio al menos dos semanas después de la cirugía, con una herida sana, bien cicatrizada, y sin material de sutura presente.

d. Antilúpicos (micofenolato, ciclosporina, azatioprina, tacrolimus):

Son potentes inmunosupresores. Su uso estará condicionado por la severidad del cuadro. En casos leves, se recomienda su suspensión al menos una semana previo a la cirugía, y reiniciarlos cinco a siete días posterior a ésta, siempre y cuando no existan complicaciones de la herida. Si existe compromiso sistémico, el riesgo de complicaciones médicas al suspenderlos supera el de la infección, por lo que se deben mantener. No obstante, en un paciente con un cuadro severo y activo, la decisión de realizar una cirugía electiva debe ser discutida con el reumatólogo y considerar diferir la cirugía, si fuese posible.

\section{Planeamiento preoperatorio: ¿cómo clasificar el protrusio y cómo posicionar los componentes?}

La planificación preoperatoria es crucial para anticipar posibles dificultades técnicas, seleccionar implantes, y tener retroalimentación intraoperatoria de la correcta ejecución de ésta. Recomendaciones acerca de los aspectos técnicos del planeamiento han sido ya ampliamente publicadas. ${ }^{12,13}$ Existen alteraciones patoanatómicas que son características en los pacientes con protrusio acetabular, y que deben considerarse al momento de planeamiento.

I. Migración medial o superomedial de la cabeza femoral:

Las clasificaciones de protrusio acetabular son descriptivas y no determinan ningún valor terapéutico. El proceso clave es restablecer el COR para lo cual existen diversos métodos. El más conocido es el del Ranawat et al., ${ }^{14}$ en el cual se calcula el $20 \%$ de la altura de la pelvis $(\mathrm{APv})$ para construir un triángulo rectángulo isósceles, cuyos lados idénticos se proyectan desde $5 \mathrm{~mm}$ lateral a la base de la "gota de Köhler" hacia proximal, y luego desde ese punto hacia lateral. Al centro de la hipotenusa se establece el COR nativo (-Figura 1). Diversos autores han cuestionado la exactitud de este método, afirmando que conlleva una ubicación proximal y medial, ${ }^{15,16}$ publicándose recientemente algunas fórmulas que estiman su ubicación en relación a la base de la gota con menor desviación que los métodos previos: ${ }^{17}$

- Hombres: $\mathrm{APv} \times 0,16$ lateral / $\mathrm{APv} \times 0,07$ proximal;

- Mujeres: $\mathrm{APv} \times 0,155$ lateral $/ \mathrm{APv} \times 0,065$ proximal.

Nuestra recomendación es posicionar la copa durante la planificación de manera anatómica, idealmente tocando, con el polo de la hemiesfera, la línea ilioisquiática. La cavidad acetabular secundaria al defecto de la protrusión puede ser rellenada con injerto óseo.

Una de las formas para predecir el tamaño adecuado de la copa es utilizar softwares para planificación digital, con la que 


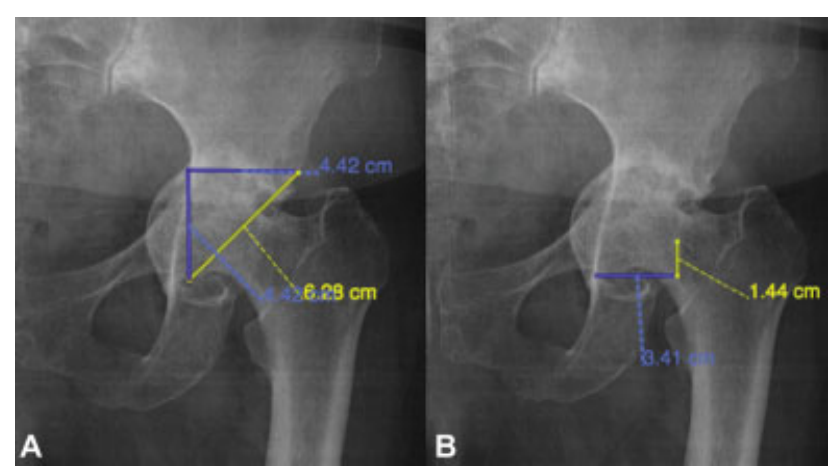

Fig. 1 Protrusio acetabular en una mujer de 83 años. Altura pélvica: $21,92 \mathrm{~cm}$. (A) Ubicación del centro de rotación de la cadera según el método de Ranawat y (B) según el método publicado por Fujii et al. ${ }^{17}$ Nótese la ubicación más medial y proximal con el método $A$.

hemos tenido buena experiencia, pero con costo asociado (-Figura 2). Otra forma es utilizar el método de plantillas de acetato sobre la pantalla del computador, nuestro actual método de elección. ${ }^{18} \mathrm{Si}$ la radiografía digital no dispone de marcadores, otro método plausible es el de los tres puntos. ${ }^{19}$

II. Acortamiento de la extremidad, pérdida de offset y tensión de partes blandas:
Dada la migración superomedial de la cabeza femoral, existe un acortamiento de la extremidad comprometida, al mismo tiempo que una disminución del offset global de la cadera. La ubicación correcta del COR trasladará éste en dirección inferior y lateral, recuperando la tensión del aparato abductor y alargando la extremidad. Otro punto importante es la frecuente presencia de coxa vara, lo cual debe ser tomado en consideración durante la planificación, dado que la mayoría de los vástagos femorales tiene un ángulo cérvico diafisiario (ACD) mayor que el de la cadera vara, generando un alargamiento de la extremidad. Durante la planificación, también resulta importante reconocer la presencia de una prominencia medial excesiva (overhang) del trocánter mayor, también conocida como lateroversión del trocánter mayor, ${ }^{20,21}$ muy frecuente en la coxa vara.

Tomando en consideración todo lo mencionado, se puede usar la planificación preoperatoria para tener retroalimentación intraoperatoria, para reconocer y enmendar posibles errores, entre ellos:

- Fresas acetabulares mayores a lo planificado: sugiriendo medialización exagerada, en la que la cavidad es mayor que el reborde, o un crecimiento excesivo que comprometa la indemnidad de las paredes acetabulares.

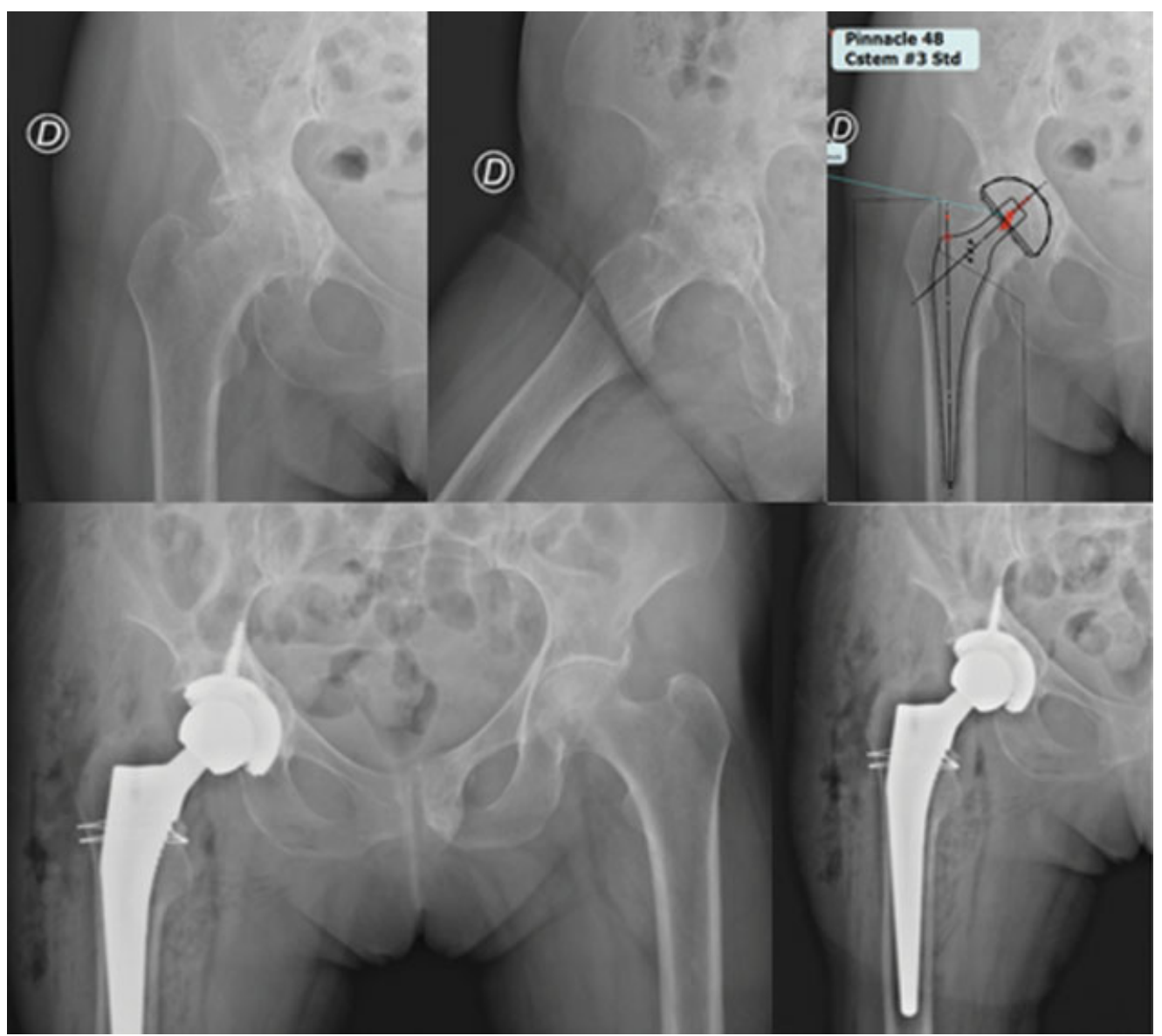

Fig. 2 Planificación preopratoria de paciente con protrusio acetabular. Radiografía postoperatoria con resolución según lo planificado. 


\section{- Raspa femoral definitiva significativamente menor que lo planificado: probable entrada y raspado en varo, con "atascamiento" de la punta de la raspa en la cortical lateral de forma prematura. Considerar también deseje en el plano sagital. \\ - Necesidad de usar un cuello de prueba lateralizado y/o necesidad de cabezas de prueba de más de $5 \mathrm{~mm}$ de diferencia para lograr tensión de partes blandas, estabilidad, $y$ rango de movimiento (ROM) sin pinzamiento: sugiere medialización inadvertida del cotilo, con pérdida de offset acetabular.}

Se debe considerar el uso de fluoroscopía intraoperatoria, ante la ocurrencia de cualquier desviación del plan preoperatorio.

4. Detalles técnicos a considerar durante el abordaje, con énfasis en el abordaje posterior

Independiente de cuál sea el abordaje a utilizar, la movilización del fémur se verá limitada por la migración medial de la cabeza femoral y la rigidez de la articulación. Además, los reparos anatómicos habituales pueden verse distorsionados.

Utilizando un abordaje posterior, se realiza una incisión centrada en el trocánter mayor, desde aproximadamente $3 \mathrm{~cm}$ proximal al trocánter hasta $6 \mathrm{~cm}$ a $8 \mathrm{~cm}$ distal a la cresta del vasto. La liberación de la inserción del glúteo mayor en el fémur (hasta $1,5 \mathrm{~cm}$ ) es un gesto que realizamos de regla en estos pacientes, evaluando las ramas de la primera arteria perforante. ${ }^{22}$ Una vez identificado el tendón del piriforme, puede ser tomado con un punto de sutura, de la misma forma que los rotadores, y separado de la cápsula. La cápsula también es liberada y tomada por uno o dos puntos de sutura; sin embargo, es altamente posible que, una vez finalizado el procedimiento, como el offset de la cadera va a cambiar sustancialmente, estas estructuras no puedan ser reinsertadas a través de puntos transóseos o a partes blandas, como es nuestra elección. Es posible también liberar la cabeza refleja del recto y la cápsula hacia anterior para tener mejor visualización.

Una vez observada la superficie del cuello femoral y distinguida del reborde posterior del acetábulo se confirma la calcificación del labrum en la mayoría de los casos. Removemos siempre alrededor de $5 \mathrm{~mm}$ de reborde acetabular posterior y superior asumiendo que es el vestigio de labrum calcificado, con un osteotomo curvo. Movilizamos la cadera para tener un mejor ángulo de observación y evaluar la posibilidad de una luxación segura, que no cree una fractura en la pared posterior del acetábulo. Es posible asistirse con un gancho óseo. Si el cirujano estima un riesgo alto al intentar realizar la luxación, hacemos una osteotomía in situ, la que puede ser con un corte simple o doble ("anillo de servilleta"). Para los casos de protrusio siempre utilizamos una hoja de sierra más angosta que la habitual. Se puede utilizar, según la disponibilidad, una sierra reciprocante. Una vez realizada la osteotomía, frecuentemente tenemos que repasar el corte femoral. Un corte de "cuello largo" puede complicar significativamente la visualización del acetábulo en estos casos. Extraemos la cabeza idealmente con un tirabuzón. No deben usarse osteótomos e intentar fragmentar el remanente de la cabeza dentro del acetábulo pues esa fuerza puede fracturar, por transmisión, el delgado trasfondo acetabular.

Otras alternativas potencialmente útiles durante el abordaje es una osteotomía trocantérica triplanar de Trousdale, $^{23}$ o la modificación de Iyer $^{24,25}$ del abordaje posterior.

\section{Preparación del acetábulo}

Para estimar el tamaño del componente acetabular, además del plan preoperatorio, intraoperatoriamente, hemos utilizado fresas tomadas por una pinza Kocher para tratar de estimar el diámetro del acetábulo a nivel del reborde antes del fresado. En general, nuestra recomendación es iniciar el fresado con una fresa de tamaño hasta $4 \mathrm{~mm}$ menor que el diámetro del reborde acetabular en estos pacientes, cuyas paredes son habitualmente delgadas. Fresas más pequeñas pueden ser utilizadas en el fondo acetabular, con discreción y cuidado, con el objetivo de crear un lecho sangrante.

Dado el defecto medial en el fondo acetabular y la formación de una especie de reloj de arena, en que el reborde acetabular funciona como el centro y luego la cavidad se expande, es posible cometer el error de medialización y crecimiento exagerado durante el fresado, con el consiguiente adelgazamiento de las paredes acetabulares. Es por esto que el fresado debe ser anatómico, buscando un press-fit ecuatorial, evitando el proceso descrito como reaming-related medialization para no comprometer el offset acetabular. ${ }^{26}$ Lateralizar el COR también debe evitarse, dado que aumenta las fuerzas de reacción articular, el desgaste, y la tasa de revisión. ${ }^{27} \mathrm{El}$ ascenso del COR es menos frecuente, aunque puede darse en casos de defectos superomediales.

La literatura describe varias técnicas para alcanzar un adecuado press-fit cuando se emplean copas no cementadas, desde un underreaming de $2 \mathrm{~mm}$, hasta un reaming de $1 \mathrm{~mm}$ de línea a línea. ${ }^{28-30}$ En nuestra experiencia utilizamos $1 \mathrm{~mm}$ de underreaming con una copa completamente hemisférica y no elíptica técnica validada en la literatura para el implante que utilizamos. ${ }^{30-32}$ En nuestro caso, el componente de prueba es considerado imprescindible para estos pacientes, para asegurarse que la cantidad de injerto a utilizar en el fondo es adecuada. La abducción y la anteversión de la copa las verificamos con la técnica de Meftah-Ranawat. ${ }^{33}$

6. Elegir entre una copa no cementada, cementada, o de metal poroso: ¿qué dice la literatura?

Históricamente, el uso de copas cementadas tuvo un éxito significativo en pacientes con protrusio acetabular, por lo que ciertamente son una gran opción en manos entrenadas. ${ }^{34-37}$ El uso de copas no cementadas, como es la práctica más frecuente en nuestro país, también tiene un resultado exitoso respaldado en la literatura. ${ }^{29-31}$ La serie más relevante fue publicada por el grupo de la Clínica Mayo, ${ }^{38}$ 


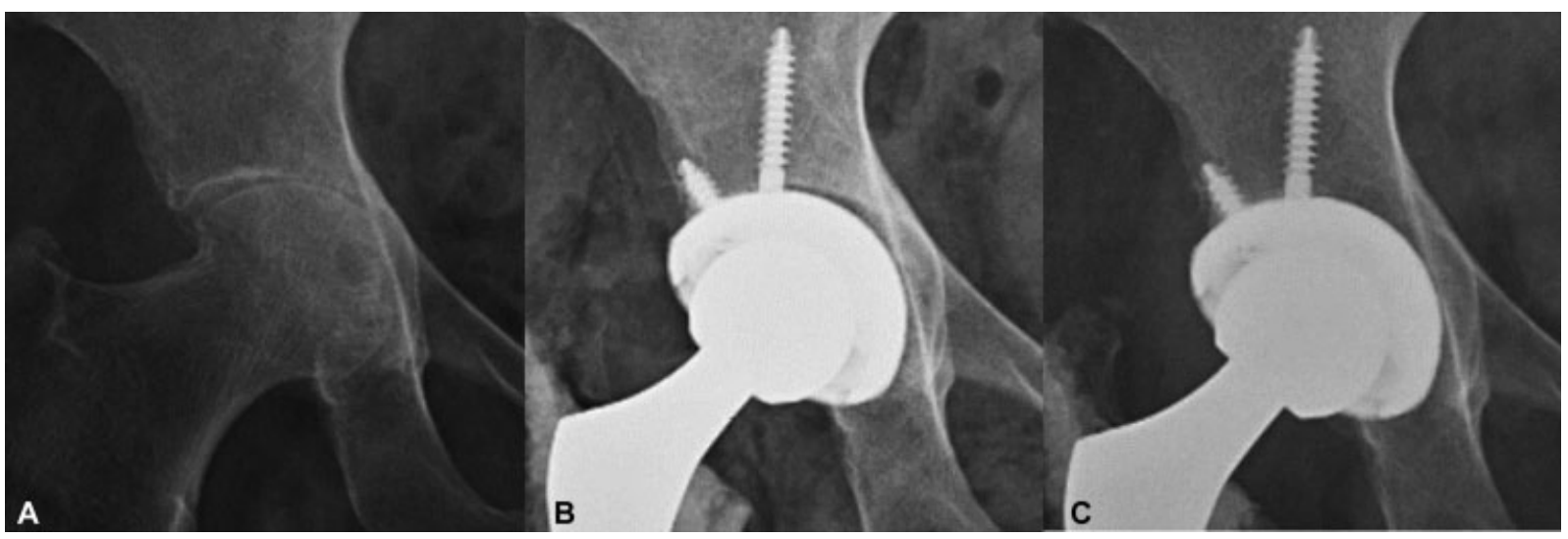

Fig. 3 (A) Imagen preoperatoria de paciente con protrusio acetabular. (B) Radiografía en el postoperatorio inmediato, con restauración del centro de rotación de la cadera e injerto en el fondo acetabular. (C) Radiografía a los tres meses, que evidencia incorporación del injerto y formación de hueso denso.

que demostró $85 \%$ de sobrevida de copas no cementadas. Es destacable que el $40 \%$ de estas copas fueron posicionadas sin tornillos, confiando absolutamente en el press-fit alcanzado durante la cirugía. Entre de las copas más usadas en esta serie están Pinnacle (DePuy, Raynham, MA, EEUU) y Trilogy (Zimmer Biomet, Warsaw, IN, EEUU), copas disponibles en nuestro país.

Las copas de metal trabecular teóricamente podrían tener ventajas en casos de primarias difíciles. Un estudio reciente ${ }^{39}$ en China demuestra $100 \%$ de sobrevida a 4,5 años utilizando copas de metal trabecular en pacientes con protrusión y artritis reumatoide. Sin embargo, un estudio ${ }^{40}$ basado en los registros suecos y australianos pone una nota de precaución acerca de un modelo en particular, Trabecular Metal (Zimmer Biomet), debido a una mayor tasa de revisión de este implante en comparación a otras copas no cementadas. Parece ser una opción razonable con la limitante de su mayor costo.

7. ¿Qué tipo de injerto uso en el fondo, y cómo lo preparo?

El uso de injerto acetabular en pacientes con protrusio acetabular ha sido pregonado desde la década de 1980 . Heywood $^{41}$ utilizó injerto sólido en 9 pacientes, con integración del $100 \%$ y buena evolución clínica. Crowninshield et al. ${ }^{42}$ propusieron que la restauración del COR acetabular disminuye el estrés en la pared medial, disminuyendo el riesgo de fallo. Posteriormente, Ranawat y Zahn ${ }^{21}$ dieron mayor soporte al concepto de restauración del COR usando injerto medial. En 23 pacientes (27 caderas), utilizando injerto óseo, lateralizaron el componente acetabular, normalizaron el COR y suplementaron la pared medial. En el mismo trabajo, ${ }^{21}$ formularon recomendaciones para el uso de injerto: cuando la protrusión es menor que $5 \mathrm{~mm}$ y la pared medial es razonablemente fuerte, no recomendaron el uso de injerto; cuando es mayor que $5 \mathrm{~mm}$ y la pared medial es delgada pero intacta, recomendaron el uso de injerto. En caso de deficiencia manifiesta de la pared medial, recomendaron el uso de injerto y consideración de métodos adicionales de suplementación. ${ }^{21}$

Las opciones a utilizar son autoinjerto, aloinjerto o sustitutos óseos. El autoinjerto de cabeza femoral es la alternativa más utilizada, por su disponibilidad. Autoinjertos sólidos han sido propuestos de distintas formas con buenos resultados; ${ }^{21,41}$ sin embargo, el uso de autoinjerto morcelizado es habitualmente de elección, por su reproducibilidad, altas tasas de consolidación del injerto y formación de hueso denso ${ }^{43}$ ( - Figura 3 ). Nuestra técnica es tomarlo directamente desde la cabeza femoral utilizando fresas de $38 \mathrm{~mm}$ en la mesa de la arsenalera.

Los resultados del uso de injerto con implantes cementados y no cementados son excelentes a corto, mediano y largo plazos. Utilizando copas cementadas, la sobrevida a 12 años ha sido reportada entre un $90 \%$ y un $94 \%$, mientras que, a 20 a 28 años, se ha reportado una sobrevida de $73 \%{ }^{44-46}$ Mullaji y Shetty, ${ }^{30}$ utilizando injerto morcelizado de $8 \mathrm{~mm}$ a $10 \mathrm{~mm}$ con copas no cementadas, reportaron un $90 \%$ de buenos a excelentes resultados a 4.2 años, con $100 \%$ de incorporación del injerto. Baghdadi et al. ${ }^{38}$ reportaron un $83 \%$ de sobrevida acetabular a 15 años, utilizando una copa no cementada e injerto morcelizado.

La adición de vancomicina en polvo ( $1 \mathrm{~g}$ mezclado con el injerto) pudiera ser una práctica razonable basada en evidencia que no está directamente relacionada a reconstrucciones en protrusio. ${ }^{47}$ Ciertamente, queda a discreción del cirujano. Nuestro grupo la ha utilizado en ocasiones en que hemos considerado el caso como de alto riesgo de complicación infecciosa.

Una vez preparado el injerto, lo posicionamos con una pinza especialmente diseñada, y lo compactamos in situ con la bola impactora del polietileno. Con el injerto impactado, evaluamos que la cantidad es suficiente posicionando la última fresa usada en la preparación del cotilo.

8. ¿Qué vástago elijo, y que detalles anatómicos son especiales en la reconstrucción femoral de pacientes con protrusio acetabular?

La elección del vástago femoral dependerá de tres variables:

I. Calidad ósea: habitualmente se trata de pacientes usuarios de corticoides crónicos, con edad promedio 
entre 65 y 70 años, con predominio del género femenino, ${ }^{14}$ lo que puede inclinar hacia el uso de vástagos cementados. Se debe observar la morfología del fémur proximal (clasificación de Dorr et al.: ${ }^{48} \mathrm{~A}, \mathrm{~B}$ o C), junto con mediciones objetivas, como el "calcar-tocanal ratio" o el "cortical thickness index", según lo descrito por Dorr et al. ${ }^{48}$

II. ACD: ante la presencia de coxa vara, la elección del implante podrá inclinarse por vástagos con opción de ACD disminuido, a modo de recrear de mejor manera el largo y el offset, aunque en la mayoría de los diseños actuales son mayores que $125^{\circ}$.

Normalmente, al lateralizar y distalizar el COR del cotilo de vuelta en su posición correcta, se aumenta el offset global y la tensión del aparato abductor, por lo cual la pérdida de offset femoral con los vástagos disponibles actualmente se ve compensada, y es habitual no requerir vástagos con offset extendido o lateralizado.

III. Prominencia medial del trocánter mayor u overhang: tiende a desplazar la entrada de las fresas iniciales hacia medial, lo que puede llevar al raspado en varo. Al mismo tiempo, intentar evitar la prominencia habitualmente posterolateral puede llevar a una entrada anterior $\mathrm{y}$ deseje en el plano sagital (recurvatum).

Los vástagos cementados tienen menor tolerancia al varo que los no cementados, ${ }^{49,50}$ producto de la generación de un manto de cemento adelgazado a nivel del calcar, con el consiguiente riesgo de fractura y aflojamiento.

La literatura no es categórica acerca de qué tipo de vástago es superior en pacientes con protrusio acetabular. Sin embargo, si se extrapola la información de estudios comparando cementados y no cementados en pacientes con artritis reumatoide, no se encuentran diferencias entre ambos tipos. $^{51}$

Las estrategias para sobrellevar estas alteraciones incluyen:

- Osteotomía del cuello femoral baja, según la planificación, para evitar alargamiento de la extremidad y utilizar vástagos femorales con $\mathrm{ACD}$ varo $\left(<125^{\circ}\right)$.

- Posicionar el osteótomo de cajón inicial suficientemente lateralizado, incluso a través del trocánter mayor.

- Cargar las raspas hacia posterior y lateral.

- Uso de cureta/cucharilla o raspa curva para lateralizar el canal a nivel del trocánter mayor. Existen sistemas que incluyen raspas cilíndricas trocantéricas para estos propósitos.

- Emplear diseños de vástagos femorales con reducción de su hombro superolateral.

9. Manejo postoperatorio: ¿carga total inmediata o lo protejo?, ¿controles clínicos y radiológicos postoperatorios?

La rehabilitación y el momento de iniciar la carga de la cadera operada está condicionada por los resultados intraoperatorios. Si la fijación es satisfactoria, en los trabajos en que usan injerto morcelizado en el fondo, con un componente acetabular no cementado, la indicación de cuándo iniciar la carga completa es variable. Distintas series $^{29,39}$ han indicado carga diferida por los primeros días e incluso semanas, carga parcial, o carga sin restricciones. Sin embargo, no existe evidencia de que alguna de estas estrategias interfiera en la integración del injerto. Por otro lado, en el trabajo de Rosenberg et al., ${ }^{46}$ indicaron reposo en cama por seis semanas y luego carga parcial por tres meses, también con buenos resultados. Nuestra recomendación es permitir carga a tolerancia con ayudas técnicas, de acuerdo con la necesidad, si hay en el intraoperatorio una fijación satisfactoria, que se complemente con una radiografía acorde del postoperatorio inmediato.

La frecuencia de los controles radiográficos no es un asunto zanjado. En su serie, Zhen et al. ${ }^{39}$ recomiendan radiografías en el postoperatorio inmediato, a las seis semanas, luego a los tres meses, a los seis meses, y, posteriormente, anual. En la serie de Zuh et al., ${ }^{29}$ indicaron control clínico y radiográfico a los tres meses y, luego, anualmente. Ambas series reportan buenos resultados. Además, distintos trabajos ${ }^{29,40}$ muestran tiempos diferentes de integración del injerto, que va entre los 4,5 meses al año. Por lo tanto, durante el primer año, es recomendable realizar controles frecuentes, entre otras cosas, para evidenciar la integración del injerto.

Nuestra práctica es realizar control radiográfico y clínico en el postoperatorio inmediato, a las seis semanas, seis meses (momento en que el injerto debiera estar integrado), al año y, luego, anualmente. Además, un control clínico a las tres semanas del postoperatorio.

10. ¿Debo esperar la misma sobrevida de los implantes que el resto de los pacientes?

La sobrevida del implante depende de múltiples factores, pero, en los pacientes con protrusio acetabular, el factor modificable central corresponde a la adecuada restitución latero-medial del COR de la cadera. Baghdadi et al. ${ }^{27}$ demostraron, en 162 pacientes con protrusio acetabular, que, por cada $1 \mathrm{~mm}$ de desplazamiento medial o lateral del COR nativo, el riesgo de revisión aumentaba en un $24 \%$, teniendo una sobrevida del componente acetabular de revisión aséptica de un 89\% y 85\% a 15 años, para componentes cementados y no cementados, respectivamente.

Zuh et al. ${ }^{29}$ utilizaron injerto morcelizado más componente acetabular no cementado en 39 caderas con protrusio acetabular, con lo que no tuvieron revisiones en un seguimiento a 4,5 años, con un $100 \%$ de integración del injerto. Rosenberg et al. ${ }^{46}$ también utilizaron injerto morcelizado en 36 artroplastias totales en pacientes con artritis reumatoide $\mathrm{y}$ protrusio acetabular, con una sobrevida del implante de $90 \%$ a 12 años de seguimiento promedio. Baghdadi et al. ${ }^{38}$ usaron, en 65 pacientes, injerto morcelizado para el fondo acetabular y un componente acetabular no cementado en el $89 \%$ de los pacientes, con lo que tuvo una sobrevida libre de revisión a 15 años de $70 \%$ en 
general, 83\% para el componente femoral, y del $85,4 \%$ para el componente acetabular. Si uno compara esta sobrevida con la sobrevida libre de cualquier reoperación de $96 \%$ que tienen implantes no cementados con pares de fricción actualmente usados, es plausible plantear que la sobrevida de los implantes en protrusio sea algo menor. Sin embargo, la mayor parte de estos trabajos no utilizaron polietilenos de última generación, lo que puede influir en los resultados ${ }^{52}$.

\section{Conclusión}

El manejo de la artroplastía total de cadera en pacientes con protrusio acetabular requiere de un abordaje multidimensional que considera el pre, intra y postoperatorio. No sólo la sistematización del enfoque, sino que el conocimiento acabado y la experiencia del equipo quirúrgico son necesarios para ofrecer al paciente la mejor oportunidad de éxito.

\section{Financiación}

Los autores no tienen fuente de financiación que declarar.

\section{Conflicto de Intereses}

Los autores no tienen conflicto de intereses que declarar.

\section{Referencias}

1 Barbour JA, Binding J, Bridges M, Kelly C. Evaluation of a screening tool for inflammatory joint disease. Ann Rheum Dis 2003;62(02): 187-188. Doi: 10.1136/ard.62.2.187

2 Emara AK, Nageeb E, George J, Buttaro MA, Higuera C, Piuzzi NS. Hypovitaminosis D in lower extremity Joint Arthroplasty: A systematic review and meta-analysis. J Orthop 2020; 21:109-116. Doi: 10.1016/j.jor.2020.03.010

3 Bible MW, Pinals RS, Palmieri GM, Pitcock JA. Protrusio acetabuli in osteoporosis and osteomalacia. Clin Exp Rheumatol 1983;1 (04):323-326

4 Lin J-A, Liao C-C, Lee Y-J, Wu C-H, Huang W-Q, Chen T-L. Adverse outcomes after major surgery in patients with systemic lupus erythematosus: a nationwide population-based study. Ann Rheum Dis 2014;73(09):1646-1651. Doi: 10.1136/annrheumdis2012-202758

5 Singh JA, Inacio MCS, Namba RS, Paxton EW. Rheumatoid arthritis is associated with higher ninety-day hospital readmission rates compared to osteoarthritis after hip or knee arthroplasty: a cohort study. Arthritis Care Res (Hoboken) 2015;67(05): 718-724. Doi: 10.1002/acr.22497

6 Goodman SM, Springer B, Guyatt G, et al. 2017 American College of Rheumatology/American Association of Hip and Knee Surgeons Guideline for the Perioperative Management of Antirheumatic Medication in Patients With Rheumatic Diseases Undergoing Elective Total Hip or Total Knee Arthroplasty. J Arthroplasty 2017;32(09):2628-2638. Doi: 10.1016/j.arth.2017.05.001

7 Goodman SM, Miller AS, Turgunbaev M, et al. Clinical Practice Guidelines: Incorporating Input From a Patient Panel. Arthritis Care Res (Hoboken) 2017;69(08):1125-1130. Doi: 10.1002/ acr.23275

8 Agca R, Heslinga SC, Rollefstad S, et al. EULAR recommendations for cardiovascular disease risk management in patients with rheumatoid arthritis and other forms of inflammatory joint disorders: 2015/2016 update. Ann Rheum Dis 2017;76(01): 17-28. Doi: 10.1136/annrheumdis-2016-209775
9 Groleau C, Morin SN, Vautour L, Amar-Zifkin A, Bessissow A. Perioperative corticosteroid administration: a systematic review and descriptive analysis. Perioper Med (Lond) 2018; 7:10. Doi: 10.1186/s13741-018-0092-9

10 Grennan DM, Gray J, Loudon J, Fear S. Methotrexate and early postoperative complications in patients with rheumatoid arthritis undergoing elective orthopaedic surgery. Ann Rheum Dis 2001;60(03):214-217. Doi: 10.1136/ard.60.3.214

11 Tanaka N, Sakahashi H, Sato E, Hirose K, Ishima T, Ishii S. Examination of the risk of continuous leflunomide treatment on the incidence of infectious complications after joint arthroplasty in patients with rheumatoid arthritis. J Clin Rheumatol 2003;9(02): 115-118. Doi: 10.1097/01.RHU.0000062514.54375.bd

12 Della Valle AG, Padgett DE, Salvati EA. Preoperative planning for primary total hip arthroplasty. J Am Acad Orthop Surg 2005;13 (07):455-462. Doi: 10.5435/00124635-200511000-00005

13 Whiddon DR, Bono JV. Digital templating in total hip arthroplasty. Instr Course Lect 2008;57:273-279

14 Ranawat CS, Dorr LD, Inglis AE. Total hip arthroplasty in protrusio acetabuli of rheumatoid arthritis. J Bone Joint Surg Am 1980;62 (07):1059-1065

15 John JF, Fisher PE. Radiographic determination of the anatomic hip joint center. A cadaver study. Acta Orthop Scand 1994;65(05): 509-510. Doi: 10.3109/17453679409000901

16 Schofer MD, Pressel T, Heyse TJ, Schmitt J, Boudriot U. Radiological determination of the anatomic hip centre from pelvic landmarks. Acta Orthop Belg 2010;76(04):479-485

17 Fujii M, Nakamura T, Hara T, Nakashima Y. Is Ranawat triangle method accurate in estimating hip joint center in Japanese population? J Orthop Sci 2021 Mar;26(02):219-224. Doi: 10.1016/j.jos.2020.03.007. Epub 2020 Mar 31. PMID: 32245695

18 Petretta R, Strelzow J, Ohly NE, Misur P, Masri BA. Acetate templating on digital images is more accurate than computerbased templating for total hip arthroplasty. Clin Orthop Relat Res 2015;473(12):3752-3759. Doi: 10.1007/s11999-015-4321-y

19 Arnaout F, Dewan V, Paliobeis C. The 3-dot circle: A reliable method for safe and efficient digital templating of the acetabular component. J Orthop 2018;15(03):787-791. Doi: 10.1016/j.jor.2018.03.027

20 Wang $\mathrm{H}, \mathrm{Gu} \mathrm{J}$, Liu X, et al. Variation in greater trochanteric lateroversion: a risk factor for femoral stem varus in total hip arthroplasty. Hip Int 2020;30(01):33-39. Doi: $10.1177 / 1120700018825248$

21 Ranawat CS, Zahn MG. Role of bone grafting in correction of protrusio acetabuli by total hip arthroplasty. J Arthroplasty 1986; 1(02):131-137. Doi: 10.1016/s0883-5403(86)80051-1

22 Sur YJ, Morsy M, Mohan AT, Zhu L, Lachman N, Saint-Cyr M. The first perforating branch of the deep femoral artery: A reliable recipient vessel for vascularized fibular grafts: An anatomical study. J Plast Reconstr Aesthet Surg 2016;69(03):351-358. Doi: 10.1016/j.bjps.2015.10.024

23 Ackerman DB, Trousdale RT. Triplanar trochanteric osteotomy: a modified anterior trochanteric slide osteotomy. J Arthroplasty 2008;23(03):459-461. Doi: 10.1016/j.arth.2007.10.006

24 Iyer KM. A new posterior approach to the hip joint. Injury 1981;13 (01):76-80. Doi: 10.1016/0020-1383(81)90098-x

25 Iyer KM. Technical Note on Modified Posterior Approach to the Hip Joint. J Orthop Case Rep 2015;5(01):69-72. Doi: 10.13107/ jocr.2250-0685.260

26 Merle C, Innmann MM, Waldstein W, et al. High Variability of Acetabular Offset in Primary Hip Osteoarthritis Influences Acetabular Reaming-A Computed Tomography-Based Anatomic Study. J Arthroplasty 2019;34(08):1808-1814. Doi: 10.1016/j. arth.2019.03.065

27 Baghdadi YMK, Larson AN, Sierra RJ. Restoration of the hip center during THA performed for protrusio acetabuli is associated with 
better implant survival. Clin Orthop Relat Res 2013;471(10): 3251-3259. Doi: 10.1007/s11999-013-3072-x

28 Cruz-Pardos A, García-Rey E, García-Cimbrelo E, OrtegaChamarro J. Alumina-on-alumina THA in patients with juvenile idiopathic arthritis: a 5-year followup study. Clin Orthop Relat Res 2012;470(05):1421-1430. Doi: 10.1007/s11999-011-2046-0

29 Zuh S-G, Zazgyva A, Gergely I, Pop TS. Acetabuloplasty with bone grafting in uncemented hip replacement for protrusion. Int Orthop 2015;39(09):1757-1763. Doi: 10.1007/s00264-015-2804-9

30 Mullaji AB, Shetty GM. Acetabular protrusio: surgical technique of dealing with a problem in depth. Bone Joint J 2013;95-B(11, Suppl A)37-40. Doi: 10.1302/0301-620X.95B11.32900

31 Mullaji AB, Marawar SV. Primary total hip arthroplasty in protrusio acetabuli using impacted morsellized bone grafting and cementless cups: a medium-term radiographic review. J Arthroplasty 2007;22(08):1143-1149. Doi: 10.1016/j.arth. 2006.11.005

32 Stihsen C, Rath C, Radl R, et al. Early migration characteristics of a $180^{\circ}$ porous-coated cup with 1 -mm press fit. Arch Orthop Trauma Surg 2013;133(05):707-712. Doi: 10.1007/s00402-013-1713-x

33 Meftah M, Yadav A, Wong AC, Ranawat AS, Ranawat CS. A novel method for accurate and reproducible functional cup positioning in total hip arthroplasty. J Arthroplasty 2013;28(07):1200-1205. Doi: $10.1016 /$ j.arth.2012.09.018

34 Slooff TJ, Huiskes R, van Horn J, Lemmens AJ. Bone grafting in total hip replacement for acetabular protrusion. Acta Orthop Scand 1984;55(06):593-596. Doi: 10.3109/17453678408992402

35 Płomiński J, Kwiatkowski K. Cemented primary total arthroplasty for acetabular protrusion in patients with rheumatoid arthritis. Ortop Traumatol Rehabil 2008;10(01):26-34

36 Dutka J, Sosin P, Skowronek P, Skowronek M. Total hip arthroplasty with bone grafts for protrusio acetabuli. Ortop Traumatol Rehabil 2011;13(05):469-477. Doi: 10.5604/15093492.967224

37 Saglam Y, Ozturk I, Cakmak MF, Ozdemir M, Yazicioglu O. Total hip arthroplasty in patients with ankylosing spondylitis: Midterm radiologic and functional results. Acta Orthop Traumatol Turc 2016;50(04):443-447. Doi: 10.1016/j.aott.2016.06.010

38 Baghdadi YMK, Larson AN, Sierra RJ. Long-term results of the uncemented acetabular component in a primary total hip arthroplasty performed for protrusio acetabuli: a fifteen year median follow-up. Int Orthop 2015;39(05):839-845. Doi: 10.1007/s00264-014-2580-y

39 Zhen P, Li X, Zhou S, Lu H, Chen H, Liu J. Total hip arthroplasty to treat acetabular protrusions secondary to rheumatoid arthritis. J Orthop Surg Res 2018;13(01):92. Doi: 10.1186/s13018-0180809-y

40 Laaksonen I, Lorimer M, Gromov K, et al. Trabecular metal acetabular components in primary total hip arthroplasty. Acta Orthop 2018;89(03):259-264. Doi: 10.1080/17453674.2018. 1431445
41 Heywood AW. Arthroplasty with a solid bone graft for protrusio acetabuli. J Bone Joint Surg Br 1980;62(03):332-336

42 Crowninshield RD, Brand RA, Pedersen DR. A stress analysis of acetabular reconstruction in protrusio acetabuli. J Bone Joint Surg Am 1983;65(04):495-499

43 Padgett DE, Kull L, Rosenberg A, Sumner DR, Galante JO. Revision of the acetabular component without cement after total hip arthroplasty. Three to six-year follow-up. J Bone Joint Surg Am 1993;75(05):663-673. Doi: 10.2106/00004623-199305000-00005

44 Busch VJJF, Gardeniers JWM, Verdonschot N, Slooff TJJH, Schreurs BW. Acetabular reconstruction with impaction bone-grafting and a cemented cup in patients younger than fifty years old: a concise follow-up, at twenty to twenty-eight years, of a previous report. J Bone Joint Surg Am 2011;93(04):367-371. Doi: 10.2106/JBJS. I.01532

45 Welten MLM, Schreurs BW, Buma P, Verdonschot N, Slooff TJJH. Acetabular reconstruction with impacted morcellized cancellous bone autograft and cemented primary total hip arthroplasty: a 10- to 17-year follow-up study. J Arthroplasty 2000;15(07): 819-824. Doi: 10.1054/arth.2000.7110

46 Rosenberg WWJ, Schreurs BW, de Waal Malefijt MC, Veth RPH, Slooff TJJH. Impacted morsellized bone grafting and cemented primary total hip arthroplasty for acetabular protrusion in patients with rheumatoid arthritis: an 8- to 18-year follow-up study of 36 hips. Acta Orthop Scand 2000;71(02):143-146. Doi: 10.1080/000164700317413102

47 Peeters A, Putzeys G, Thorrez L. Current Insights in the Application of Bone Grafts for Local Antibiotic Delivery in Bone Reconstruction Surgery. J Bone Jt Infect 2019;4(05):245-253. Doi: $10.7150 /$ jbji.38373

48 Dorr LD, Faugere MC, Mackel AM, Gruen TA, Bognar B, Malluche $\mathrm{HH}$. Structural and cellular assessment of bone quality of proximal femur. Bone 1993;14(03):231-242. Doi: 10.1016/ 8756-3282(93)90146-2

49 Jaffe WL, Hawkins CA. Normalized and proportionalized cemented femoral stem survivorship at 15 years. J Arthroplasty 1999;14(06):708-713. Doi: 10.1016/s0883-5403(99)90226-7

50 Davey JR, O'Connor DO, Burke DW, Harris WH. Femoral component offset. Its effect on strain in bone-cement. J Arthroplasty 1993;8(01):23-26

51 Mäkelä KT, Eskelinen A, Pulkkinen P, Virolainen P, Paavolainen P, Remes V. Cemented versus cementless total hip replacements in patients fifty-five years of age or older with rheumatoid arthritis.J Bone Joint Surg Am 2011;93(02):178-186. Doi: 10.2106/JBJS. I.01283

52 Hopper RH Jr, Ho H, Sritulanondha S, Williams AC, Engh CAJr. Otto Aufranc Award: Crosslinking Reduces THA Wear, Osteolysis, and Revision Rates at 15-year Followup Compared With Noncrosslinked Polyethylene. Clin Orthop Relat Res 2018;476 (02):279-290. Doi: 10.1007/s11999.0000000000000036 